\title{
Tactile localization of the direction and distance of sounds
}

\author{
B. L. RICHARDSON \\ University of Toronto, Toronto, Ontario M1C 1A4, Canada \\ - and \\ B. J. FROST \\ Queen's University, Kingston, Ontario, Canada
}

\begin{abstract}
In a previous paper, experiments were reported which demonstrated that human subjects can judge accurately the azimuthal direction of sounds using a tactile localization device. It was also demonstrated that a tactile analogue of selective auditory attention was possible with this system. Three additional experiments, reported here, indicate that subjects are also able to judge the distance of the sound source and can concurrently judge both the azimuthal direction and distance of a source. Comparisons were made between conditions where head movements were permitted (active) and conditions where the head was held still (passive), and between normal auditory judgments and tactile judgments. Active tactile performance was essentially similar to auditory performance. Active performance was superior to passive in both directional and distance judgment, but different components of the motor-sensory complex were found to contribute to active superiority in the two tasks. The implication of these experiments for the design of auditory prosthetic devices is discussed.
\end{abstract}

Perhaps because speech perception occupies a position of high priority among auditory functions, most auditory prosthetic devices that present sounds to the skin have been designed to display frequency information at the expense of other sources of information which are present in the auditory signal. For example, cues that permit the localization of a sound source have hardly ever been present in such devices. This omission may have consequences more serious than the deprivation of location information per se, because the knowledge of a sound's spatial location appears to be important for the operation of some "higher" perceptual processes.

One such process has been called selective listening (Neisser, 1967), or more generally, selective auditory attention. This refers to the ability to attend to one stimulus and ignore another which is occurring at the same time and is therefore competing with the first. The value of such a process is particularly obvious when it is necessary to "listen" to one of several people who are speaking at the same time. While several variables have been shown to affect selective auditory attention, the ability to perceive competing

This research was supported in part by a grant from the Ontario Department of University Affairs and in part by National Research Council of Canada Grant AO 353 to B. J. Frost. B. L. Richardson was supported by an Ontario Mental Health Foundation Fellowship. B. L. Richardson is now at the Department of Psychology, Scarborough College, University of Toronto, 1265 Military Trail, West Hill, Ontario MIC 1A4. Requests for reprints should be sent to this address. sounds as originating from different locations is probably the most crucial (Broadbent, 1958, Neisser, 1967).

That the skin can be used for the purpose of sound localization has been demonstrated by Békésy (1955), Frost and Richardson (1976), and Gescheider (1965, 1970). In the Frost and Richardson study, normal hearing was masked and miniature microphones were placed over the subject's ears. The outputs of these microphones were independently amplified and fed to two vibrators, upon which subjects rested their fingertips. Tactile judgments of sound direction (in the horizontal plane) compared favorably with those of normal audition. Furthermore, an analogue of selective auditory attention was demonstrated in which subjects were able to discriminate the location of concurrent sounds whose direction of origin was separated by less than $5^{\circ}$ azimuth angle (Richardson, Wuillemin, \& Saunders, 1978).

In normal audition, head movements create binaural intensity differences which are constantly changing in a manner lawfully related to those head movements and the position of the sound source in auditory space. Freedman and Fisher (1968) and Wallach $(1939,1940)$ have shown that localization performance depends, in many circumstances, upon the information derived from such interaction with the environment. Head movements were also found to improve directional accuracy when judgments depended upon tactile information alone (Frost \& Richardson, 1976). In addition, it has been suggested that "externaliza- 
tion," or the ability to perceive an object as "out there" rather than existing at the receptor site, depends upon observer-induced changes in sensation such that these changes are lawfully related to the position of the distal stimulus and the movements of the observer (Wallach, 1938; White, Saunders, Scadden, Bach-y-Rita, \& Collins, 1970). Subjects using the tactile localization device described above reported sensations similar to the "phantom" sensations described by Békésy (1959). These sensations were reported to pass from one locus of vibration to the other as the head rotated from side to side.

It appears, therefore, that there are several parallels between the mechanism of auditory directionality and those operative when the skin is used for the same purpose. However, true localization involves the judgment of direction and distance, so a more meaningful assessment of the skin's potential for sound localization should include a comparison of tactile and auditory distance judgments.

While there has been little systematic study directed towards determining the means by which auditory distance judgments are made, it is clear that there are a number of potential cues listeners may use. Examples of these are echos (Supa, Cotzin, \& Dallenbach, 1944; Worchel \& Dallenbach, 1947), loudness (Békésy, 1949), and timbre (Békésy, 1960; Woodworth \& Schlosberg, 1955).

When a sound source is located somewhere off the medial plane, the difference between the distances to the two ears is called the binaural distance difference. Because sound pressure decreases with propagation distance, the nearer of the two ears will receive a more intense signal than the further. This is so irrespective of, and in addition to, the head's sound shadow effect (Gulick, 1971), which not only causes a reduction of sound intensity at the far ear but also reduces the high-frequency content of the signal it receives (Fedderson, Sandell, Teas, \& Jeffress, 1957). These effects presumably combine to produce an overall binaural difference cue to directionality and, within a limited range, to distance also. When a sound is located directly at the opening to one of the ears, the binaural differences are at a maximum. The sound has to travel practically no distance to reach the nearer ear and half the circumference of the head to reach the further ear. Moreover, the sound shadow effect is also at its maximum. As the sound moves further from the head, both these effects must decrease. The difference in the distance which the sound has to travel to reach the two ears remains roughly the same, but, at further distance, the binaural distance difference represents only a small proportion of the total distance the sound has traveled and the decrease in intensity due to propagation to the further ear compared to the nearer ear will be less (Gulick, 1971). Similarly, the amount of diffraction or "bending around the head" that is necessary to reach the fur- ther ear also decreases. These differential effects as a function of source distance combine to represent another possible source of information for distance judgments.

When a sound source is located in the subject's medial plane and the head is then rotated back and forth between the $0^{\circ}$ and $180^{\circ}$ azimuth angle, there will result changes in the intensity of sound impinging on the ears such that they alternately receive a maximum and minimum intensity. The range of these maxima and minima will presumably decrease as the sound source moves further away from the head. Thus, the rate of change of intensity for a given arc through which the head is rotated will also decrease at greater distances of the sound source. In audition, there are binaural temporal cues which can contribute to judgments of sound distance. However, it appears unlikely that tactile judgments can be made on the basis of such temporal information (Gescheider, 1970). Which cues are operative in various circumstances is difficult enough to determine, but harder still is the task of assessing the relative contribution of these sources of information when, as must often be the case, the final percept is multidetermined. Nevertheless, one of the objects of the experiments reported here was to investigate the stimulus parameters upon which judgments of distance were likely to have been made.

In the first experiment, the accuracy of tactile and auditory distance judgments was compared under active (head movements permitted) and passive (head held still) conditions. In the second experiment, the subjects were required to judge the direction and distance of a sound stimulus. This task more closely corresponded to localization in normal perceptual conditions.

\section{EXPERIMENT 1: TACTILE JUDGMENTS OF THE DISTANCE OF SOUNDS}

\section{Method}

Subjects. Two male and two female psychology students, whose ages ranged from 21 to 33 years, served as subjects in these experiments.

Apparatus. Two miniature electret condenser microphones (Thermo Electron, Model 5333) were mounted on the outside of the earpieces of a pair of stereophonic headphones (Sharpe, Model HA8). The outputs of the microphones were independently amplified by a Sony TC 252 stereophonic tape recorder. Channel 1 of the tape recorder was connected with a $4-\mathrm{ohm}, 5-\mathrm{cm}$-diam speaker, the paper cone of which had been removed. A $1.5-\mathrm{cm}$ diam rounded Teflon tip was glued to the end of the speaker coil, and the subject's left index finger rested upon this tip. Channel 2 of the tape recorder was connected to a similarly modified speaker which served as a vibrotactile stimulator upon which the subject's right index finger rested.

The sound to be located was presented by an $8-0 h m, 10-\mathrm{cm}$-diam loudspeaker, level with and directly in front of the subject's binaural axis. The speaker was suspended from a $90-\mathrm{cm}$ boom, the center of rotation of which was directly above the subject's head (see Figure 1).

The stimulus (delivered by a Grass S8 stimulus generator) con- 


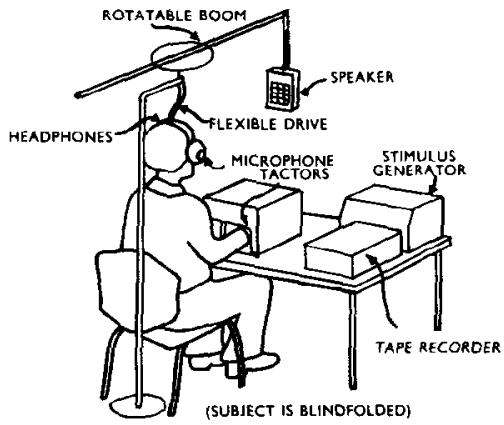

Figure 1. Diagram of apparatus used in Experiments 1, 2, and 3.

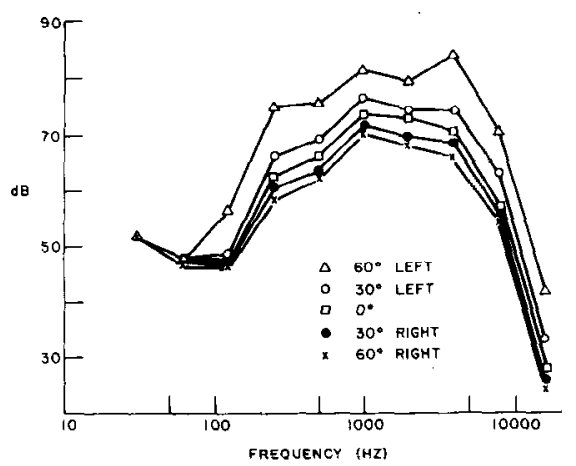

Figure 2. Sound pressure level (decibels re .0002 dynes $/ \mathrm{em}^{2}$ ) at the various frequencies of stimulus energy as a function of different directions of the sound source. Readings were taken from the left microphone output when the stimulus was positioned $17.5 \mathrm{~cm}$ from the center of the subject's binaural axis.

sisted of .3-msec, $30-\mathrm{V}$ pulses in trains of $90 \mathrm{msec}$ duration (eight pulses per train). The trains were presented at a rate of $6 / \mathrm{sec}$, and the phenomenal sound was a "buzz" of complex spectral composition. The stimulus signal was also delivered diotically to the subject's headphones, where it produced a sound which totally masked normal auditory localization (for data confirming this, see Frost \& Richardson, 1976). A potentiometer, wired into a Wheatsone bridge circuit, was connected to the headphones' headband by a flexible drive shaft. The circu it was arranged so that $360^{\circ}=3.6 \mathrm{~V}$, which allowed head rotation to be read directly from a digital voltmeter to an accuracy of $\pm 1^{\circ}$.

A Brüil and Kjaer spectral analyzer was used to determine decibel values (re .0002 dynes $/ \mathrm{cm}^{2}$ ) at the various frequencies present in the stimulus signal when the stimulus sound was $45 \mathrm{~cm}$ from the microphone.

The analysis showed that decibal values increased steadily from approximately 50 to $85 \mathrm{~dB}$ between 20 and $1,000 \mathrm{~Hz}$, beyond which signal energy decreased until, at $15,000 \mathrm{~Hz}$, no energy was recorded (see Frost \& Richardson, 1976, for pen recordings of spectral analyzer output).

Sound-pressure-level (SPL) readings were taken with a Brüel and Kjaer Type 2204 sound-level meter equipped with an octave filter set with a range from 31.5 to $16,000 \mathrm{~Hz}$. The headphones, upon which the microphones were mounted, were placed on a styrofoam "dummy" head, and the amplified output of the left microphone (normally the input to the left vibrator) was connected to a soundinsulated headphone which forms the input section of the Type 4152 Brüel and Kjaer artificial ear. The device fits directly onto the sound-pressure meter and replaces the microphone normally used for taking SPL readings in open-field conditions.

Using this apparatus, it was possible to take direct readings of a microphone's response to variations of SPLs as a function of stimulus distance (the three distances used were $17.5,45$, and $80 \mathrm{~cm}$ ) from the subject's binaural axis and azimuth angle (which varied by intervals of $10^{\circ}$ from $60^{\circ}$ left to $60^{\circ}$ right of the subject's medial plane).

With the head facing directly forward $\left(0^{\circ}\right.$ azimuth $)$ and the loudspeaker positioned at the $17.5 \cdot \mathrm{cm}$ distance, the SPL was recorded for the 10 frequencies shown in Figure 2. The first 10 recordings were taken with the speaker at $60^{\circ}$ to the right, and the process was then repeated for the other azimuth angles shown in Figure 2. Figure 3 shows the average SPL (measured across all frequencies) at various angles of stimulus azimuth and the three stimulus distances. Since the portion of the energy spectrum to which the skin is most sensitive lies below $1,000 \mathrm{~Hz}$ (Békésy, 1959), the SPL readings between 125 and $1,000 \mathrm{~Hz}$ were recorded for stimuli at 17.5- and $80-\mathrm{cm}$ distances. The differences between these values were calculated and Figure 4 shows these values as a function of azimuthal location of the stimulus. ${ }^{1}$

Procedure. The subjects' accuracy of distance judgment was tested under four conditions: active auditory, passive auditory, active tactile, and passive tactile. The subjects were blindfolded in all four conditions. In the auditory condition, the subjects wore the headband section of the headphones in order to permit measurement of head rotation, and judgments were made on the basis of normal audition. In the tactile condition, hearing was masked and judgments were made with tactile information alone. The active condition permitted head movements, but the subjects were given no instructions concerning the time they should take to respond. Passive trials consisted of holding the head still while facing directly ahead until the 5-sec stimulus had ended, at which

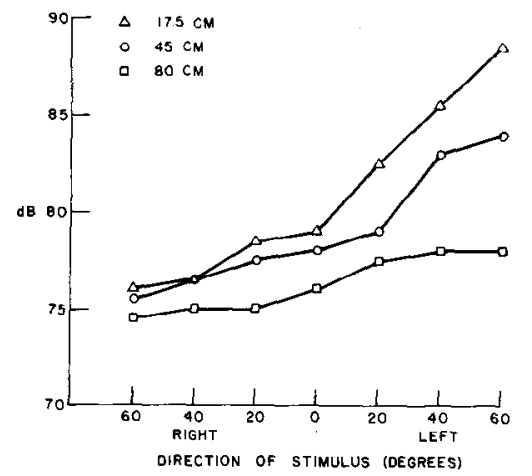

Figure 3. Averaged sound pressure level (i.e., averaged across all frequencies between 31.5 and $16,000 \mathrm{~Hz}$ ) as a function of stimulus direction and distance from the center of the subject's bingural axis.

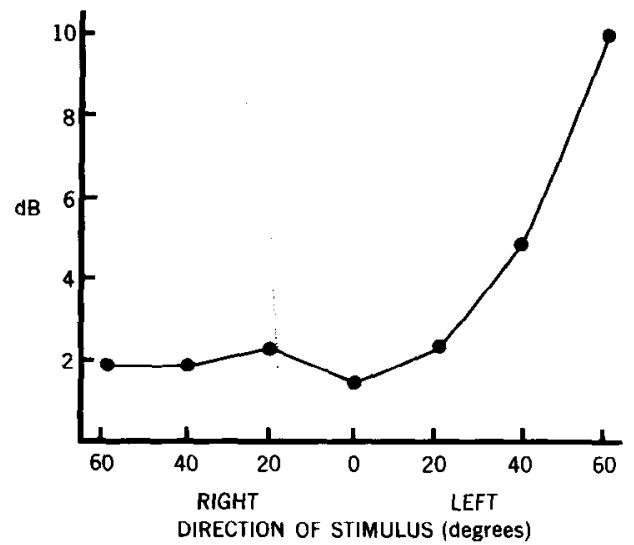

Figure 4. Intensity differences (SPL, re $\mathbf{. 0 0 0 2}$ dynes $/ \mathrm{cm}^{2}$ averaged across 125, 250, 500, and $1,000 \mathrm{~Hz}$ ) between stimuli at 17.5- and $80-\mathrm{cm}$ distances as a function of azimuth angle. 
Table 1

Total and Mean Errors of Each Subject for the 18 Distance Judgments made in Each of the Two Modalities and Two Conditions in Experiment 1

\begin{tabular}{cccccc}
\hline & \multicolumn{2}{c}{ Auditory } & & \multicolumn{2}{c}{ Tactile } \\
\cline { 2 - 3 } \cline { 5 - 6 } Subject & Passive & Active & & Passive & Active \\
\hline 1 & 1 & 2 & 3 & 1 \\
2 & 1 & 0 & 4 & 0 \\
3 & 3 & 0 & 5 & 0 \\
4 & 3 & 0 & 5 & 1 \\
Total & 8 & 2 & & 17 & 2 \\
Mean Error & 2 & .5 & & 4.25 & .5 \\
p(Binomial) & $<.001$ & $<.001$ & $<.01$ & $<.001$ \\
\hline
\end{tabular}

Note-Stimulus distances were $17.5,45$, and $80 \mathrm{~cm}$ from the center of the subjects' binaural axis. Probability of means equal to or smaller than those obtained are shown at the bottom of the table.

time subjects were required to make a response. Each subject received 18 trials ( 6 at each of the three distances) in the two modalities and two conditions. Thus, every subject made a total of 72 distance judgments, and at the end of each trial, a verbal report of "near," "medium," or "far," was required. The stimuli were presented at the three distances in random order and, when errors occurred, the subjects were informed of the position of the speaker.

\section{Results}

Table 1 shows the total and mean errors of each subject for the 18 trials under each of four conditions. Performance under the four combinations is well above chance level responding, as determined by the binomial probability distribution with $\mathrm{N}=18$, $\mathrm{p}=1 / 3$, and $\mathrm{q}=2 / 3$.

Active responding is equally good for both auditory and tactile judgments of distance, and passive performance, while inferior to active, is more obviously inferior in the tactile modality. A repeatedmeasures analysis of variance of the data is presented in Table 2; it shows that auditory performance is superior to tactile $(p<.05)$ and active superior to passive $(p<.05)$.

The significant interaction between modality and condition $(p<.05)$ suggests that the poorer tactile passive performance is the major contributor to the main effects.

\section{Discussion}

This experiment indicates that a tactile sound localization device can be used for the purpose of detec- ting sound distance and that the accuracy with which this can be done approximates normal auditory performance. There appears to be a clear superiority of active performance over passive, and while this is the case for both modalities, it is particularly obvious in the tactile modality. The similarity of performance in the active conditions of both modalities is most encouraging, since auditory distance judgments are probably cued by temporal and intensity information, while tactile cues are likely to be restricted to intensity information alone (Gescheider, 1965, 1970). In the interest os optimizing tactile display design, it would be useful to consider what potential information differences exist which may account for the superiority of active judgments.

In the passive condition of this experiment, in which the stimulus was positioned at $0^{\circ}$ azimuth, the only likely tactile cue to distance was the absolute intensity difference between the three stimuli. As Figure 3 shows, the average SPL difference for stimuli at $17.5,45$, and $80 \mathrm{~cm}$ was between 1 and $2 \mathrm{~dB}$.

However, these values are derived from measures of SPL at frequencies which, though within the ear's range of resonsiveness, lie outside the skin's range of maximal sensitivity. Figure 4 shows that within the frequencies to which the skin is most sensitive, the recorded stimulus intensity at $17.5 \mathrm{~cm}$ differs from that at $80 \mathrm{~cm}$ by about $2 \mathrm{~dB}$ or less when the stimulus is located at $0^{\circ}$ azimuth or to the right of the subject's medial plane (and the microphone involved in the recording is on the left side of the head).

The situation is altogether different for the active condition. As shown in Figures 3 and 4, with the stimulus at $60^{\circ}$ to the left (which could obviously be achieved with appropriate head movements regardless of the position of the stimulus), the maximum intensity difference value is increased to about $10 \mathrm{~dB}$.

These measurements suggest that one reason for superior active tactile discrimination is that head movements increase the difference of tactile stimulus intensity at the two fingertips. The measurements also suggest that tactile discrimination of distance may by possible when the stimuli differ in intensity by as little as $1 \mathrm{~dB}$ SPL. (Mills, 1958, has reported that the minimal audible binaural intensity difference is about $.5 \mathrm{~dB}$ ). In general, the data presented in Figure 2 support the conclusions derived from

Table 2

Repeated Measures Analysis of Variance of Errors in Judgment of Distance in the Two Modalities and Two Conditions of Experiment 1

\begin{tabular}{|c|c|c|c|c|c|}
\hline Source & df & SS & MS & $\mathbf{F}$ & $\mathbf{p}$ \\
\hline Subjects & 3 & 2.19 & .73 & & \\
\hline Modality & 1 & 5.06 & 5.06 & 22.0 & $<.05$ \\
\hline Condition & 1 & 27.56 & 27.56 & 11.53 & $<.05$ \\
\hline Modality by Condition & 1 & 4.98 & 4.98 & 19.92 & $<.05$ \\
\hline Modality by Subjects & 3 & .69 & .23 & & \\
\hline Condition by Subjects & 3 & 7.19 & 2.39 & & \\
\hline Modality by Condition by Subjects & 3 & .77 & .25 & & \\
\hline
\end{tabular}

Note-The distances used were $17.5,45$, and $80 \mathrm{~cm}$ from the center of the subjects' binaural axis. 
measurements displayed in Figures 3 and 4, since the intensity differences created by head rotation follow the same general pattern in all three figures.

There are other possible reasons for the superiority of active performance. For example, as can be seen in Figures 3 and 4, rate of change of intensity for a given angle of stimulus rotation might also serve as a cue for discriminating these stimuli. It should also be remembered that Figures 2, 3, and 4 pertain to intensity changes at the left microphone alone. The inverse of these changes occurs at the right microphone. The simultaneous occurrence of these changes could be an extra source of tactile information.

The main contribution of head movements seems to be that they create discriminable pattern differences that are not available in the passive case, at least when the stimulus is in the medial plane. The next experiment was concerned with the judgments of distance at a number of different azimuth angles and required a response which indicated the perceived azimuthal direction of the sound source at the same time.

\section{EXPERIMENT 2: DIRECTION AND DISTANCE JUDGMENTS COMBINED}

The tactile display is capable of providing information about a sound's azimuthal direction and distance simultaneously. The relative intensity of vibration (or position of the single felt locus) at the fingertips and correlated "knowledge of head position" permit decisions of direction to be made. The overall or absolute intensity and, with head movements, the rate of intensity changes referred to in Experiment 1 furnish distance information. Thus, in one display, information concerning both parameters is simultaneously transmitted to the skin, fulfilling the necessary conditions for two-dimensional localization of a sound source. (Pinna cues responsible for vertical plane localization are, of course, absent in this system at present, but, since most significant stimuli encountered in a normal auditory environment originate from a limited range in the vertical dimension, this limitation seems minor.)

This experiment was designed to measure the accuracy with which subjects can use this tactile sound localization device to locate a sound in two dimensions.

\footnotetext{
Method

Subjects. The subjects used for this experiment were the same as those who served in Experiment 1.

Apparatus and Procedure. Essentially, the procedure followed that used in Experiment 1. However, in each trial in this experiment, the speaker was positioned in one of the 13 directions, at $10^{\circ}$ intervals between $60^{\circ}$ left and $60^{\circ}$ right of the subject's medial plane. The speaker was also positioned at one of three distances along these axes, the values being identical to those used in Experiment 1 (i.e., 17.5, 45, and $80 \mathrm{~cm}$ from the center of the subject's binaural axis).
}

Thus, a total of 39 trials of randomly assigned combinations of the 13 directions and 3 distances were given under each of the auditory and tactile modalities and active and passive conditions. In the active condition, the subjects indicated the perceived direction of the sound by bringing their heads to rest facing in that direction and verbally reported the distance of the sound as "near," "medium," or "far." In the passive condition, the subjects were required to point their heads in the direction from which the sound had appeared to come after it had been terminated. This response was then followed with a verbal report of "near," "medium," or "far." As was the case for Experiment 1, feedback was given for distance judgments but feedback was not given for directional judgments.

Table 3

Mean Errors of Directional Judgments at the Three Distances, Two Modalities, and Two Conditions of Experiment 2

\begin{tabular}{lcccccc} 
& \multicolumn{2}{c}{ Auditory } & & \multicolumn{2}{c}{ Tactile } & \\
\cline { 2 - 3 } & Passive & Active & & Passive & Active & $\overline{\mathrm{X}}$ Error \\
\hline Near & 5.12 & 4.89 & 11.79 & 5.15 & 6.73 \\
Medium & 5.64 & 4.83 & & 13.06 & 6.46 & 7.49 \\
Far & 6.46 & 5.56 & 13.53 & 6.43 & & 8.86 \\
$\bar{X}$ Error & 5.74 & 5.09 & 13.53 & 6.43 & $\overline{\bar{X}}=7.69$ \\
\hline
\end{tabular}

Note-Mean errors in degrees; distances, $17.5,45$, and $80 \mathrm{~cm}$; modalities, auditory and tactile; and conditions, passive and active.

Table 4

Summary Table of Four-Factor Analysis of Variance of Errors in Judgment of Direction During Test of Localization in Experiment 2

\begin{tabular}{|c|c|c|c|c|c|}
\hline Source & SS & df & MS & $\mathbf{F}$ & $\mathbf{p}$ \\
\hline S & 266.32 & & & & \\
\hline $\mathbf{A}$ & 759.23 & 12 & 63.26 & & \\
\hline$A$ by $S$ & 2317.27 & 36 & 64.36 & & \\
\hline C & 2342.43 & 1 & 2342.43 & 45.49 & $<.01$ \\
\hline C by $S$ & 154.46 & 3 & 51.48 & & \\
\hline $\mathbf{M}$ & 3254.20 & 1 & 3254.20 & 20.33 & $<.05$ \\
\hline $\mathbf{M}$ by $\mathbf{S}$ & 480.08 & 3 & 160.02 & & \\
\hline D & 482.34 & 2 & 241.17 & & \\
\hline D by $S$ & 526.44 & 6 & 87.74 & & \\
\hline A by $C$ & 531.37 & 12 & 44.28 & & \\
\hline A by $C$ by $S$ & 2178.13 & 36 & 60.50 & & \\
\hline A by $\mathbf{M}$ & 857.18 & 12 & 71.43 & & \\
\hline A by $M$ by $S$ & 1793.93 & 36 & 49.83 & & \\
\hline A by $D$ & 1317.86 & 24 & 109.82 & & \\
\hline$A$ by $D$ by $S$ & 2785.50 & 72 & 38.68 & & \\
\hline C by $M$ & 1625.07 & 1 & 1625.07 & 7.47 & $<.07$ \\
\hline$C$ by $M$ by $S$ & 651.92 & 3 & 217.30 & & \\
\hline$C$ by $D$ & 31.42 & 2 & 15.71 & & \\
\hline C by $D$ by $S$ & 309.88 & 6 & 51.63 & & \\
\hline M by D & 129.50 & 2 & 64.74 & & \\
\hline$M$ by $D$ by $S$ & 203.80 & 6 & 37.96 & & \\
\hline A by C by $M$ & 277.98 & 12 & 23.16 & & \\
\hline A by $C$ by $M$ by $S$ & 1461.42 & 36 & 40.59 & & \\
\hline A by $C$ by $D$ & 1696.45 & 24 & 70.68 & & \\
\hline$A$ by $C$ by $D$ by $S$ & 3670.07 & 72 & 50.97 & & \\
\hline A by $M$ by $D$ & 1549.04 & 24 & 64.54 & & \\
\hline A by $M$ by $D$ by $S$ & 3699.48 & 72 & 51.38 & & \\
\hline C by $M$ by $D$ & 12.51 & 2 & 6.25 & & \\
\hline C by $M$ by $D$ by $S$ & 274.23 & 6 & 45.70 & & \\
\hline A by $C$ by $M$ by $D$ & 1587.36 & 24 & 66.14 & & \\
\hline$A$ by $C$ by $M$ by $D$ by $S$ & 2810.72 & 72 & 39.03 & & \\
\hline
\end{tabular}

Note-The four factors were angle (of stimulus), modality (auditory vs. tactile), condition (active vs. passive), and distance of stimulus from the center of the subjects' binaural axis. The distances used were $17.5,45$, and $80 \mathrm{~cm} . A=$ angle, $C=$ condition, $M=$ modality, $D=$ distance, $S=$ subjects. 
Table 5

Total and Mean Errors in Distance Judgments Over 39 Trials and Four Combinations of Two Modalities and Two Conditions in Experiment 2

\begin{tabular}{cccccc}
\hline & \multicolumn{2}{c}{ Auditory } & & \multicolumn{2}{c}{ Tactile } \\
\cline { 2 - 3 } \cline { 5 - 6 } Subject & Passive & Active & & Passive & Active \\
\hline 1 & 12 & 5 & 11 & 5 \\
2 & 4 & 3 & 15 & 3 \\
3 & 15 & 12 & 17 & 10 \\
4 & 4 & 4 & 8 & 9 \\
Total & 33 & 25 & & 54 & 25 \\
Mean & 8.25 & 6.25 & 13.5 & 6.25 \\
p(Binomial) & $<.001$ & $<.001$ & $<.01$ & $<.001$ \\
\hline
\end{tabular}

\section{Results}

Directional judgments. Table 3 shows the mean errors for directional judgments at the three distances under the two modalities and two conditions of Experiment 2. A four-factor repeated-measures analysis of variance of the data in Experiment 2 is summarized in Table 4 and shows that active performance is significantly better than passive $(\mathrm{p}<.01)$ and auditory better than tactile $(p<.05)$. Distance of stimulus and azimuth angle had no significant effect on directional judgments.

Distance judgments. Table 5 shows the total and mean errors in distance judgments over the 39 trials for the four subjects under the two modalities and two conditions. The probabilities associated with each of these outcomes, according to the binomial distribution with $\mathrm{N}=39, \mathrm{p}=1 / 3$, and $\mathrm{q}=2 / 3$, are shown at the bottom of the table. Again, tactile active performance is as good as auditory active. In fact, an analysis of variance of this data, summarized in Table 6, shows no significant difference between auditory and tactile performance.

Neither is there a significant difference between passive and active performance. However, the significant interaction between modality and condition $(p<.01)$ is consistent with the pattern of performance so far observed in previous analyses, namely, that poorer tactile passive performance is the major contributor to variance.

\section{Discussion}

There was, in both modalities, an increase in the proportion of distance judgment errors compared with
Experiment 1. This may have occurred because the perceived distance of the stimulus seemed to be a function of several different variables not present in Experiment 1. First, subjects of ten reported that a stimulus originating from the extreme lateral direction felt stronger (and sounded louder) than one in the medial plane. This was particularly apparent at the near distance $(17.5 \mathrm{~cm})$, for, in the passive condition, the speaker moved appreciably closer to the microphone when rotated to $60^{\circ}$ azimuth angle.

In reality, of course, the distance of the sound source from the ears is not constant for varying directions of stimulus location when the center of rotation of the boom is directly above the center of the binaural axis, as indeed it was in these experiments. Because the judgment of distance on any given trial is affected by the perceived intensity of the stimulus on the previous trial, the change of intensity when direction is varied is an obvious source of distance judgment error. Subjects often expressed surprise when, following a correct judgment of "near" for a stimulus at an eccentric azimuth angle, their judgment of "medium" for a "near" stimulus at zero azimuth was said to be incorrect. Such errors were particularly apparent in the passive condition of both modalities and are not surprising in view of the effects of direction and distance on intensity of sound at the microphone. For example, Figure 2 shows that at $0^{\circ}$ azimuth, a stimulus set at a distance of $45 \mathrm{~cm}$ yields the same SPL at the left microphone as does a stimulus positioned at $80 \mathrm{~cm}$ and $60^{\circ}$ to the left. Several other different combinations of direction and distance can be seen to create similar intensity signals and are therefore potential sources of error.

The consistency with which tactile responding, at least in the active condition, has competed favorably or equaled auditory responding throughout the experiments so far reported is noteworthy. This is perhaps surprising in view of the various advantages which audition appears to enjoy. Apart from general observations to do with the evolution of the ear as a specialized receptor, it is likely that binaural temporal cues (difference in time of arrival of sounds at the two ears) could have favored audition in these experiments. Furthermore, it is possible that at least two of the distances used in these experiments (17.5 and $45 \mathrm{~cm}$ ) were within a range in which SPL

Table 6

Summary Table of Repeated Measure Analysis of Variance of Errors in Judgment of Distance in the Four Combinations of Two Modalities and Two Conditions in Experiment 2

\begin{tabular}{lrrrrr}
\hline \multicolumn{1}{c}{ Source } & SS & df & MS & F & p \\
\hline Subjects & 109.69 & 3 & 36.56 & \\
Modality & 27.56 & 1 & 27.56 & \\
Subjects by Modality & 21.19 & 3 & 7.06 & \\
Condition & 85.56 & 1 & 85.56 & & \\
Subjects by Condition & 65.69 & 3 & 21.89 & \multirow{2}{*}{62.65} \\
Modality by Condition & 27.57 & 1 & 27.57 & .01 \\
Subjects by Modality by Condition & 1.32 & 3 & .44 & & \\
\hline
\end{tabular}


and the phase of the particle velocity might contribute to auditory judgments of sound distance (Békésy, 1960). Also of importance is the general superiority of active responding over passive, particularly during directional judgments. There may, in fact, be important differences between the contribution that head movements make in direction and distance judgments.

To begin with, "reafferent" information is of obvious importance for directional judgments. With head movements, a clear change in tactile pattern occurs as the head's direction of regard passes through the direction in which the stimulus lies. Thus, there might be two correlated inputs. One is proprioceptive information concerning head rotation (and/or a corollary discharge from the motor centers responsible for head rotation), and the other is the tactile pattern changes which are directly related to and created by those head movements. When, as the head is rotated, the direction of regard corresponds to the sound source direction, a unique tactile pattern, associated with that direction of regard, is perceived. Physically, the intensity of vibration is equal at both fingertips. Phenomenally, the "phantom sensation" (Békésy, 1959 ) is located halfway between the two fingertips. Perception (by proprioception) of the head's rotation, or of the initiating motor commands, combined simultaneously with perception of the unique tactile pattern provides for perception of the direction in which the source lies. The direction of the sound source will be the direction of the head's regard at that instant.

Without head movements, directional judgments have to be made on the basis of the relative intensity of vibration at the two fingertips. Such information is also available when the head is moving, but is of course, constantly changing in a manner lawfully related to those head movements and the position of the sound source. Static judgments can thus be seen to be based on one "frozen" temporal segment of the tactile pattern which is normally present only for an instant in the dynamic case. It is rather like stopping a movie at a single frame and, by studying it analytically, attempting to predict what is in the adjacent frames. Head movements could thus serve two functions in judgments of direction. First, they increase the "richness" of the tactile display, and second, because they are correlated with the changes in the tactile display, they permit the observer to relate those changes to a particular head position and a corresponding direction of sound source. The latter function may involve a reafferent feedback loop which assists directional accuracy.

It is not immediately apparent that head movements perform a similar function in the judgment of sound distance. Instead, it seems probable that the active condition permits distance information to be derived from the range of intensities which head rotations create. Thus, for the same amount of head rotation, the changes in intensity in tactile stimulation will be greater for nearer sounds than for distant ones, as can be seen in Figure 3 and 4. The same figures show that rate of change of intensity, as a function of head rotation, is also greater for closer stimuli. In other words, though head movements effect an improvement in distance judgment performance, they may do so for reasons which differ from those applicable to direction judgments. Experiment 3 was designed to investigate the role of head movements in distance judgments.

\section{EXPERIMENT 3: THE ROLE OF HEAD MOVEMENTS}

The question of concern in this experiment was whether or not subjects can use tactile information to judge distances of sound sources if the skin received tactile patterns identical to those created by head movements but in the absence of those head movements. If the head movements themselves are, in some way, essential for accuracy of distance judgments, then errors would be expected to increase when these head movements do not accompany the tactile patterns for which they were originally responsible.

\section{Method}

Subjects. The subjects who served in Experiments 1 and 2, also served in this experiment.

Apparatus. The apparatus used was identical to that used for Experiments 1 and 2, except that the tape recorder previously used only as a stereo amplifier was used, in this experiment, not only to amplify the outputs of the microphones, but to record on tape the signals that were being conveyed to the tactors.

Thus, as the subject's head rotated, say to the right of the sound source, the output of the left microphone increased and that of the right decreased. These fluctuations in intensity were recorded, as they occurred, on the two stereo channels of the tape recorder.

Procedure. The subjects were presented with six stimuli at each of the three distances used in Experiment 2 (18 trials in total). Following this session, called the "record session," the tape recording was played back (with the trials in a different random order from that used in the recording session) to the finger vibrators, and subjects were required to judge whether the stimulus had been in the near, medium, or far position. To mask normal hearing, the auditory stimulus was presented from the speaker, received by the microphones, and fed to the headphones during these playback trials just as it had been during the record session. However, the signals received at the microphones were no longer conveyed to the fingers; in fact, the subjects' head movements now made no difference whatever to the tactile display. Instead, the subjects received (in a different order) identical tactile patterns to those they had received as a result of their own head movements during the recording session.

In case it was possible to recognize the various tactile patterns on the basis of some idiosyncratic cue (such as the rate of the subject's own head movements), each subject was also presented with the recordings made with a different subject. The playback session was thus essentially the same as active responding in that the tactile patterns were dynamic, but the same as passive responding in that head movements had no effect on the tactile display. The stimulus complexity of active responding was present, but related headmovement information was not.

\section{Results}

Table 7 shows the number of errors for each subject performing in the active tactile condition during 
Table 7

Number of Errors for Each Subject Performing in the Active Tactile Condition During the 18 Trials of the Three Sessions in Experiment 3

\begin{tabular}{cccc}
\hline & Record & $\begin{array}{c}\text { Playback- } \\
\text { Own }\end{array}$ & $\begin{array}{c}\text { Playback- } \\
\text { Others }\end{array}$ \\
\hline $\mathrm{S}_{1}$ & 1 & 4 & 2 \\
$\mathrm{~S}_{2}$ & 1 & 1 & 2 \\
$\mathrm{~S}_{3}$ & 0 & 2 & 3 \\
$\mathrm{~S}_{4}$ & 1 & 3 & 0 \\
\hline$\overline{\mathrm{X}}$ & .75 & 2.5 & 1.75 \\
\hline
\end{tabular}

Note-The first session consisted of the recording of tactile patterns which the subjects produced themselves (record). The second session (playback-own) consisted of each subject's receiving these same recordings (in a different order) and in an essentially "passive" condition. The third session (playback-others) consisted of the presentation of the tactile patterns created by a different subject.

the 18 trials of recording and two playback sessions. All mean error outcomes were significantly better than chance level performance $(p<.001)$ as determined by the binomial probability distribution for $\mathrm{N}=18$, $p=1 / 3$, and $q=2 / 3$. The slight increase in number of errors in the two playback conditions proved nonsignificant when compared (by $t$ test for dependent samples) with the record sessions $(p>.05)$.

\section{Discussion}

The results of Experiment 3 suggest that head movements do not have to accompany the tactile patterns which they create for performance in the "active" condition to be maintained. The results also suggest that, for this task anyway, the superiority of active over passive performance does not depend upon the principle of reafference. It appears instead that active performance is better than passive, because, in the active condition, the dynamic tactile pattern contains more information upon which to base perceptual decisions.

There remains the possibility that head movements are of initial importance in "making sense" of the tactile pattern changes for which those movements are responsible. That is, performance in the two playback sessions of Experiment 3 may have depended, in part, on experience gained during the record session when head movements did accompany the tactile sensations. Although there was no test of these possisibilities, it seems unlikely that head movements assisted in the interpretations of the tactile patterns they created (and that this experience somehow carried over to the playback conditions). Experience during the record session, if an important determinant of performance, would be expected to favor discriminations of tactile stimuli most closely resembling the "training" patterns. The small difference between performance with tactile patterns created by the subject's own head movements and those created by others' head movements was in the reverse direction of that predicted by a view which attributes success in playback sessions to "experience" in the record session.

\section{GENERAL DISCUSSION}

The results of these experiments suggest that the skin can make use of stimulus intensity differences which have originated from sounds whose intensities may differ by as little as $1 \mathrm{~dB}$ SPL. However, with the measures taken in this experiment, it is not possible to specify that the minimum intensity difference used in tactile judgments was as little as $1 \mathrm{~dB}$, since this figure is an average of the differences in energy existing at various frequencies (albeit within the skin's "sensitivity" range) and these differences were sometimes larger than $1 \mathrm{~dB}$, at least at one of the frequencies included within the average. Though head movements were responsible for the production of these intensity differences (and, as a consequence, the superiority of active performance), the role of head movements in directional and distance judgments seems to differ.

For distance judgments, head movements did not appear to serve any function beyond that of producing a tactile pattern containing more information than the pattern present in the passive condition. For directional judgments, the relationship between head position and the associated tactile pattern was crucial, though the relative importance of proprioceptive information (knowledge of head position) vs. motor information (corollary discharge) could not be determined.

In normal environmental conditions, the sounds to be localized are much more variable and complex than those used as stimuli in this series of experiments, and, unlike the blindfolded subjects in these experiments, users of the tactile device would be locating sounds in the presence of powerful visual cues which would, at the very least, reduce the number of places from which a sound could be expected to come. Furthermore, information about what the sound is (temporal cues, etc.) would largely determine its likely location. Thus, the conditions under which subjects performed in these experiments were considerably more impoverished than those normally encountered. While useful for the control of variables, observations about the importance of head movements in such circumstances may be misleading, for their role in the formation of multidetermined percepts in complex environments may be quite different.

From a practical standpoint, the results of these experiments are encouraging since tactile performance compared favorably with that of normal audition, in which sound localization is a demonstrably important process. It is suggested, on the basis of these results, that the benefits which normal audition derives from sound localization are likely to apply to a tactile communication system which includes sound localization information. 


\section{REFERENCES}

BÉx́́sY, G. v. On the resonance curve and decay period at various points on the cochlea partition. Journal of the Acoustical Society of America, 1949, 21, 245.254.

BÉkÉsY, G. v. Human skin perception of travelling waves similar to those on the cochlea. Joumal of the Acoustical Society of America, 1955, 27, 830-884.

BÉKÉsY, G. v. Similarities between hearing and skin sensations, Psychological Review, 1959, 66, 1-22.

BÉKÉsY, G. v. Experiments in hearing. New York: McGraw-Hill, 1960.

Broadbent, D. E. Perception and communication. New York: Pergamon Press, 1958.

Fedderson, W. E., Sandell, T. T., Teas, D. C., \& Jeffress, L. A. Localization of high frequency tones. Joumal of the Acoustical Society of America, 1957, 29, 988-991.

Freedman, S. J., \& Fisher, H. G. The role of the pinna in auditory localizations. In S. F. Freedman (Ed.), The neuropsychology of spatially oriented behavior. Homewood, Ill: Dorey, 1968.

Frost, B. J., \& Richardson, B. L. Tactile localization of sounds: Acuity, tracking moving sources, and selective attention. Journal of the Acoustical Society of America, 1976, 59, 907-914.

GESCHEIDER, G. A. Cutaneous sound localization. Journal of Experimental Psychology, 1965, 70, 617-625.

GESCHEIDER, G. A. Some comparisons between touch and hearing. IEEE Transactions on Man-Machine Systems, 1970, MMS-11, 28-35.

Gulick, W. L. Hearing. London: Oxford University Press, 1971.

Mruss, A. W. On the minimum audible angle. Journal of the Acoustical Society of America, 1958, 30, 237-246.

NeIsser, U. Cognitive psychology. New York: Appleton-CenturyCrofts, 1967.

Richardson, B. L., Wuillemin, D. B., \& Saunders, F. Tactile discrimination of competing sounds. Perception \& Psychophysics, 1978, 24, 546-550.

Supa, M., Cotzin, M., \& Dallenbach, K. M. Facial vision. American Journal of Psychology, 1944, 47, 133-183.

Wallach, H. Über die Wahrnehmung der Schallrichtung. Psychologische Forschung, 1938, 22, 238-266.

WALLACH, H. On sound localization. Joumal of the Acoustical Society of America, 1939, 10, 270-274.

WALLACH, $H$. The role of head movements and vestibular and visual cues in sound localization. Joumal of Experimental Psychology, 1940, 27, 339-368.
White, B. W., SAunders, F. A., Scadden, L., Bach-y-Rita, P., \& Collins, C. C. Seeing with the skin. Perception \& Psychophysics, 1970, 7, 23-27.

WOODWORTH, R. S., \& SCHLOSBERG, H. Experimental psychology. London: Methuen, 1955.

Worchel, P., \& Dallenbach, K. M. Facial vision. Perception of obstacles by the deaf-blind. American Journal of Psychology, $1947,60,502-553$.

\section{NOTES}

1. Since the transmission of the stimulus from stimulus generator to the skin's surface involved several transfer functions, the stimulus measures reported here do not necessarily reflect values of the proximal stimulus. Also, it was considered desirable to allow subjects to vary fingertip position and pressure so that optimal stimulus "clarity" could be achieved. Such variations necessarily affected the proximal stimulus in a manner resistant to prediction or measurement. However, nonspecification of the proximal stimulus does not prevent meaningful comparisons of performance within the various conditions of these experiments, since there is no reason to suppose that the transfer functions of the equipment or subject finger placement and pressure resulted in proximal stimuli variations which selectively favored one condition over another.

2. All subjects reported that "something" seemed to move from one finger to the other as the head was rotated back and forth. When one finger received an increasing intensity of stimulation, the phantom sensation was perceived to move towards that finger. During active judgments, subjects attended to the apparent position of the sensation as it moved across the space between the two fingers. The direction of the sound source corresponded to the direction in which the head was facing when the locus of the phantom was felt to be halfway between the two fingertips. When head movements ceased, the sensation was reported to become more diffuse or to disappear. As the phantom disappeared, subjects became more aware of the veridical stimulation.

(Received for publication December 27, 1977; revision accepted January 2, 1979.) 